\title{
VARIAÇÃO DE PESO, GRAU DE ESCOLARIDADE, SANEAMENTO BÁSICO, ETILISMO, TABAGISMO E HÁBITO ALIMENTAR PREGRESSO EM PACIENTES COM CÂNCER DE ESTÔMAGO
}

\author{
Lidiane Pereira MAGALHÃES, Celina Tizuko Fujiyama OSHIMA, Lessileia Gomes SOUZA, \\ Jacqueline Miranda de LIMA, Luciana de CARVALHO e Nora Manoukian FORONES
}

RESUMO - Racional - Cerca de 35\% dos casos de câncer estão envolvidos com fatores advindos da dieta e de outros como o álcool, o fumo, a luz solar, agentes químicos e infecções por vírus. O câncer de estômago é o segundo tipo de câncer mais diagnosticado no mundo, sendo responsável por cerca de 9,9\% de todos os diagnósticos e cerca de 12,1\% das mortes. Objetivos - Comparar o peso corpóreo atual e habitual, grau de escolaridade, saneamento básico, tabagismo, etilismo e freqüência pregressa de consumo de alimentos entre pacientes com câncer de estômago e população controle. Método - Setenta pacientes com câncer gástrico foram pareados a outros 70 indivíduos sem diagnóstico de neoplasia. Foram coletados peso, altura, informações sobre grau de escolaridade, saneamento básico, tabagismo e etilismo e preenchido um questionário de freqüência alimentar pregressa. Resultados - Dos 75 pacientes elegíveis para o estudo, 42 eram homens, com média de idade de 59,5 anos. O grupo de pacientes com câncer apresentou menor peso e índice de massa corporal atual do que o grupo controle. Em relação ao grau de escolaridade, verificou-se que, no grupo de pacientes com câncer, 21 indivíduos nunca ingressaram na escola e dos que estudaram, cerca de $55 \%$ não conseguiram concluir o primeiro grau. Nesse grupo, 32,9\% dos indivíduos tiveram acesso ao saneamento básico e 37,1\% à eletricidade no passado; no grupo controle, esse percentual foi de $68,6 \%$ para cada uma das variáveis. No grupo caso, $58,6 \%$ dos pacientes moraram na zona rural, enquanto no grupo controle apenas $7,1 \%$. No grupo caso, $65.7 \%$ eram fumantes versus $44.3 \%$, no grupo controle. Observou-se também que os pacientes com câncer fumaram por período maior. $\mathrm{O}$ alcoolismo também foi mais freqüente entre os pacientes com câncer (45\% versus 19\%). Verificou-se que, entre os pacientes com câncer, houve maior consumo de alimentos ricos em sal, condimentos, nitratos, gordura animal saturada, carboidratos complexos, açúcar refinado e salgados fritos, comparado ao grupo controle. Conclusão - Os doentes com câncer gástrico, quando comparados ao grupo controle, apresentaram: perda de peso, queda da qualidade de vida verificada pelo baixo acesso a saneamento básico, eletricidade e escolaridade, residiram predominantemente em área rural, alta incidência de alcoolismo e alta ingestão de alimentos ricos em gorduras, alimentos industrializados e álcool.

DESCRITORES - Neoplasias gástricas. Alterações do peso corporal. Escolaridade. Saneamento básico. Alcoolismo. Tabagismo. Hábitos alimentares.

\section{INTRODUÇÃO}

O câncer, segundo dados da Organização Mundial da Saúde, é diagnosticado em cerca de 11 milhões de pessoas por ano no mundo. Estudos sugerem que essa doença poderá atingir cerca de 16 milhões por ano em $2020^{(29)}$. Anualmente, cerca de 7 milhões de mortes são provenientes de câncer, o que representa $12,5 \%$ do total de mortes no mundo ${ }^{(29)}$. Os cânceres de pulmão, colorretal e de estômago são os tumores mais comuns no mundo para homens e mulheres ${ }^{(29)}$.

Indivíduos que apresentam câncer do sistema digestório relatam freqüentemente perda de peso em curto período de tempo ${ }^{(1,2)}$, dificuldade de alimentação, dor local, náuseas, vômitos, flatulência, sensação de plenitude precoce que contribuem para o agravamento da doença, dificultam os tratamentos propostos e, conseqüentemente, favorecem pior prognóstico ${ }^{(1,2,16)}$.

O câncer de estômago é o segundo mais diagnosticado no mundo, sendo responsável por cerca de $9,9 \%$ de todos os diagnósticos e cerca de $12,1 \%$ das mortes. Sua incidência é maior em algumas regiões da China, Ásia e América do $\mathrm{Sul}^{(29)}$.

Cerca de 35\% destes cânceres estão relacionados a fatores advindos da dieta ${ }^{(16,19,25,30)}$, assim como do consumo de álcool, do uso de tabaco, da exposição à luz solar, de agentes químicos e infecções por vírus ${ }^{(9)}$. 
A partir desses dados da literatura, procurou-se traçar um perfil da população atendida no ambulatório de Oncologia da Disciplina de Gastroenterologia da Universidade Federal de São Paulo - UNIFESP/EPM, São Paulo, SP, buscando-se informações sobre variação de peso, grau de escolaridade, saneamento básico, eletricidade, condições de moradia, etilismo, tabagismo e hábito alimentar pregresso, a fim de verificar se esses doentes utilizavam fatores protetores ou promotores do câncer, comparando-se a indivíduos que não apresentavam histórico de neoplasia.

\section{MÉTODO}

Foram analisados 70 doentes com câncer gástrico (grupo caso), atendidos no ambulatório de Oncologia da Disciplina de Gastroenterologia da Universidade Federal de São Paulo, no período de março de 2004 a julho de 2005. Os pacientes do grupo caso foram selecionados de acordo com o diagnóstico anatomopatológico de adenocarcinoma gástrico, independente do estádio clínico da doença, e pareados a 70 indivíduos do mesmo sexo e idade (com variação máxima de 5 anos), atendidos em outros ambulatórios da mesma instituição no mesmo período e que constituíram o grupo controle.

A presente pesquisa foi aprovada pelo Comitê de Ética em Pesquisa da UNIFESP/EPM. Todos os pacientes concordaram em participar do estudo e assinaram termo de consentimento.

Foram estabelecidos os seguintes critérios de inclusão para os dois grupos: idade entre 30 e 90 anos, ter condições clínicas adequadas para responder às perguntas do questionário e para aferir peso e altura.

Foi aplicada uma entrevista e preenchido um questionário onde constavam pedidos de informações de dados pessoais (nome completo, idade, sexo e estado civil, registro hospitalar, endereço e telefone, para a localização do indivíduo). Foram também solicitadas informações sobre o grau de escolaridade, local de residência atual e de 20 anos atrás (capital, interior, sítio ou litoral) e se tem ou teve acesso a saneamento básico e luz elétrica. Sobre tabagismo, foi mensurada a quantidade de cigarros por dia e sobre etilismo, foi calculada a quantidade em gramas de álcool consumida por dia, de acordo com os valores referidos por LARANJEIRA e PINSKY ${ }^{(7)}$, em 1998. Foi mensurado o tempo de fumo e de consumo de álcool.

Foi realizada a coleta da medida de peso e altura e dos pacientes com câncer de estômago, foram coletadas informações sobre o estádio clínico, e se foi ou não realizado algum procedimento cirúrgico.

Elaborou-se, para este estudo, um questionário considerandose os alimentos mais freqüentemente consumidos pela população brasileira, baseado no questionário utilizado por SACHS ${ }^{(22)}$. Esses alimentos foram classificados em seis grandes grupos, para facilitar a entrevista:

grupo 1 = alimentos de origem animal que são fontes de proteínas, colesterol e gorduras saturadas, submetidos a cocção ou não;

grupo 2 = alimentos de origem vegetal que são fontes de fibras, vitaminas e minerais, submetidos a cocção ou não;

grupo 3 = alimentos fonte de gorduras, sendo de origem animal ou vegetal; grupo 4 = alimentos fonte de carboidratos simples ou complexos e gorduras;

grupo 5 = alimentos utilizados como temperos de preparações; grupo 6 = bebidas alcoólicas ou não-alcoólicas e infusões.

\section{Análise estatística}

As variáveis quantitativas foram analisadas através do cálculo de médias e desvios-padrão e as variáveis qualitativas, através das freqüências absolutas e relativas. Para análise da hipótese de igualdade de médias entre dois grupos, utilizou-se o teste $t$ de Student e, quando a suposição de normalidade dos dados foi rejeitada, utilizou-se o teste não-paramétrico de Mann-Whitney ${ }^{(21)}$. Para se testar a homogeneidade dos grupos em relação às proporções, foi utilizado o teste do qui-quadrado ou o teste exato de Fisher (quando ocorreram freqüências esperadas abaixo de 5) ${ }^{(21)}$. O nível de significância utilizado para os testes foi de $5 \%$.

\section{RESULTADOS}

Entre os 70 indivíduos do grupo caso, 42 (60\%) eram homens e a idade média encontrada foi de 60,10 anos, variando entre 34 e 82 anos. O grupo controle foi também constituído por 42 pacientes do sexo masculino, com média de idade de 59,70 anos (variação: 36-81 anos) (Tabela 1).

Dos 70 pacientes, 64 (92\%) foram submetidos a procedimento cirúrgico (Tabela 2) e 61 (95,3\%) apresentaram alterações dos hábitos alimentares após a cirurgia (Tabela 3). Todos os doentes foram operados há mais de 6 meses. $\mathrm{Na}$ análise da extensão

TABELA 1. Idade e sexo dos pacientes dos dois grupos

\begin{tabular}{lcc}
\hline & Grupo-caso & Grupo-controle \\
\hline Sexo & 42 & 42 \\
masculino & 28 & 28 \\
feminino & & \\
Idade & 60,10 & 59.70 \\
média & $36-81$ & $34-82$ \\
variação & 9,32 & 11,08 \\
DP & & \\
\hline
\end{tabular}

TABELA 2. Distribuição e freqüências dos tipos de cirurgia realizadas nos pacientes com câncer gástrico

\begin{tabular}{lcc}
\hline Tipo de cirurgia & $\mathrm{n}$ & $\%$ \\
\hline Gastrectomia subtotal & 42 & 65,6 \\
Gastrectomia total & 17 & 26,6 \\
Gastroenteroanastomose sem ressecção do tumor & 5 & 7,8 \\
\hline
\end{tabular}

TABELA 3. Alterações dos hábitos alimentares ocorridas após a cirurgia dos 61 pacientes submetidos a cirurgia para o tratamento do câncer gástrico

\begin{tabular}{lcc}
\hline Tipo de alteração & $\mathbf{n}$ & $\%$ \\
\hline Redução do volume alimentar & 29 & 47,5 \\
$\begin{array}{l}\text { Redução do volume e alteração } \\
\text { na consistência }\end{array}$ & 13 & 21,3 \\
$\begin{array}{l}\text { Redução do volume e aumento } \\
\text { do fracionamento }\end{array}$ & 12 & 19,7 \\
Voltou a se alimentar & 7 & 11,5 \\
\hline
\end{tabular}


do tumor, $35(50,1 \%)$ invadiam a serosa (T3) e $23(32,9 \%)$ apresentavam metástases em linfonodos adjacentes ou à distância (N1-2) (Figura 1). Todos os pacientes já haviam sido submetidos a algum dos tipos de tratamento relacionados anteriormente.

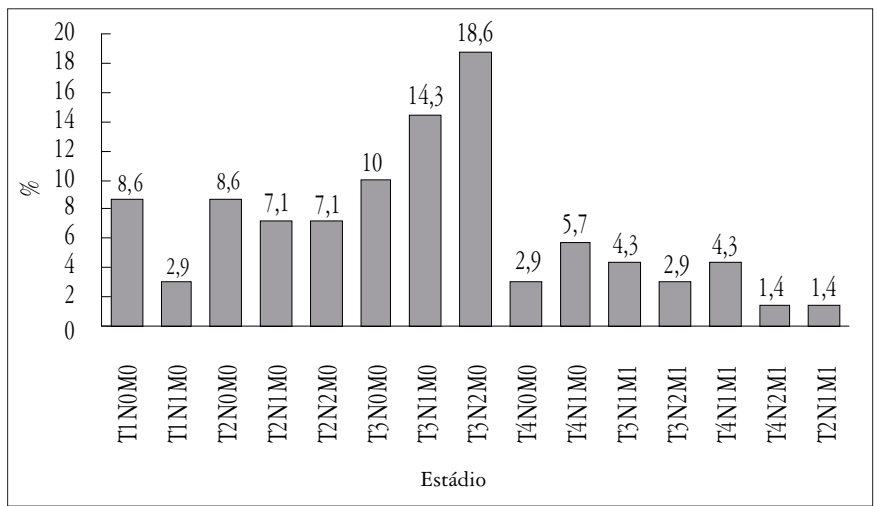

FIGURA 1. Histograma apresentando as frequiências relativas ao estádio clínico dos 70 pacientes com câncer gástrico

Observou-se que o grupo de pacientes com câncer submetidos a ressecção cirúrgica e/ou a quimioterapia e radioterapia, apresentaram valor significantemente menor de peso e índice de massa corporal (IMC) atual $(P<0,001)$ do que o grupo controle. Notou-se que os indivíduos do grupo caso apresentavam no passado, em média, o IMC em sobrepeso. Os indivíduos do grupo controle também apresentavam o IMC em sobrepeso e apresentaram, por ocasião do estudo, discreto aumento deste índice.

Em relação à escolaridade, 48 (69\%) indivíduos do grupo controle tinham concluído alguma fase do ensino, enquanto no grupo de doentes, cerca de $27(39 \%)$ atingiram o mesmo índice. Esta diferença foi estatisticamente significante, $P<0,001$ (Figura 2).

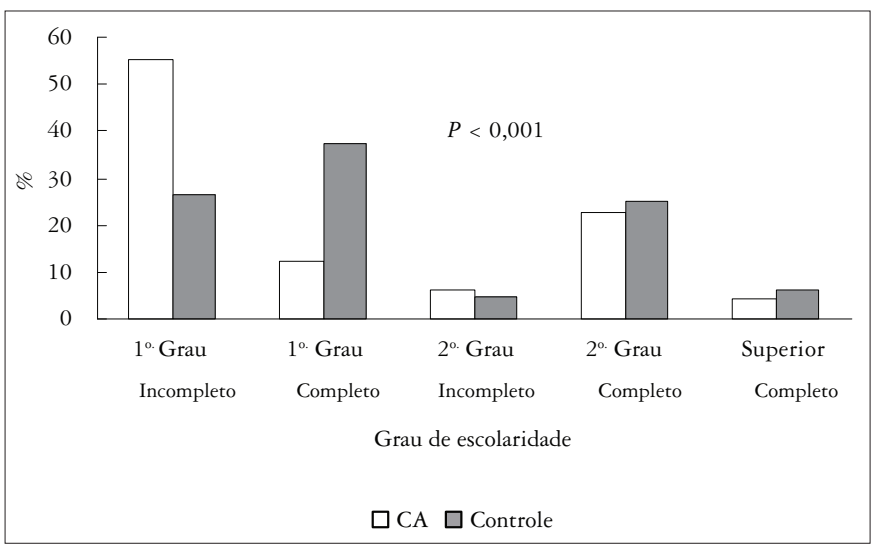

FIGURA 2. Histograma do grau de escolaridade dos pacientes com câncer gástrico (CA) e grupo controle

Observou-se que os grupos com câncer e controle não apresentaram diferença em relação à moradia, acesso ao saneamento básico e à energia elétrica no período atual, ao contrário do que foi relatado no período de 20 anos atrás. No grupo caso, 23 (32,9\%) indivíduos tiveram acesso a saneamento básico e $26(37,1 \%)$ à eletricidade no passado, enquanto que no grupo controle este percentual foi de $68,6 \%$ (48 indivíduos) para qualquer das duas variáveis $(P=0,001)$. O grupo de doentes apresentou $41(58,6 \%)$ pessoas com moradia na área rural, enquanto o grupo controle apresentou $5(7,1 \%)(P<0,001)$ (Figuras 3 e 4$)$.

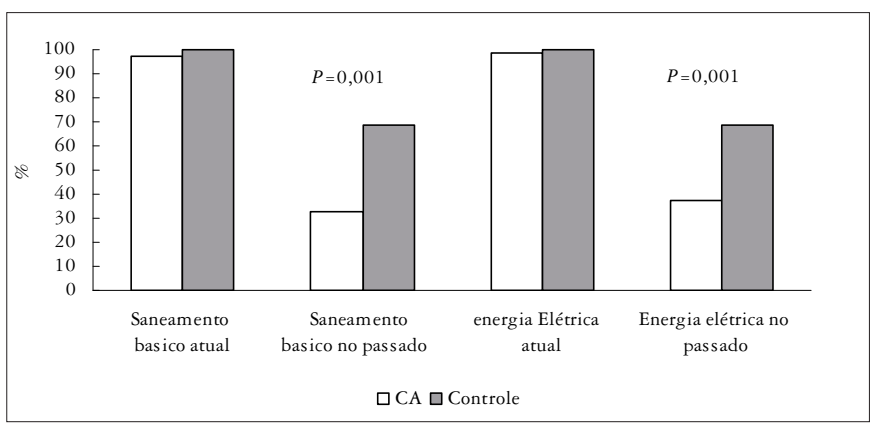

FIGURA 3. Histograma apresentando o acesso ao saneamento básico e à eletricidade dos pacientes com câncer gástrico (CA) e grupo controle

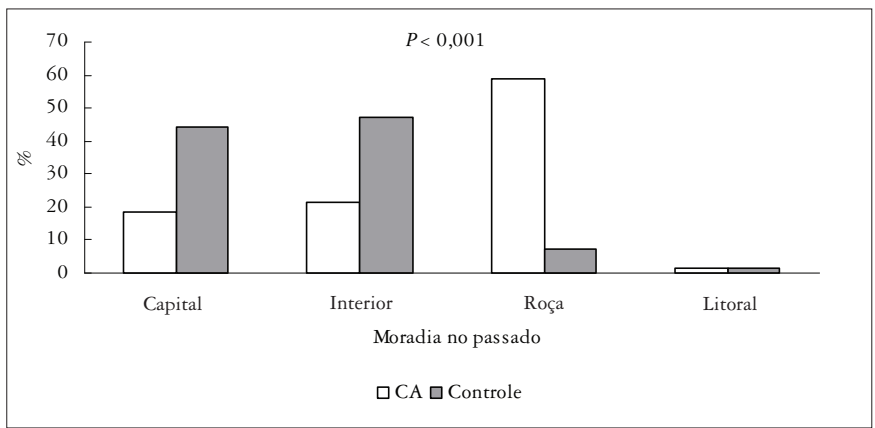

FIGURA 4. Histograma apresentando o local de moradia no passado dos pacientes com câncer gástrico (CA) e grupo controle

Dos 70 pacientes com câncer gástrico, $46(65,7 \%)$ declararam ser fumantes e no grupo controle, $31(44,3 \%)$, sendo significante essa diferença $(P=0,017)$. Porém, os grupos não diferiram em relação à quantidade de cigarros consumidos. Em relação ao tempo de consumo: os pacientes do grupo caso apresentaram média de aproximadamente 33 anos e os indivíduos do grupo controle 25 anos, diferença estatisticamente significante $(P<0,015)$. O consumo de álcool foi maior nos doentes com câncer (31 versus $13, P<0,001$ ). Não foi observada diferença no tempo de consumo de álcool.

Em relação ao consumo de alimentos de origem animal (grupo 1), observou-se que o grupo de doentes apresentou maior consumo de ovos, gemada, carne bovina frita e assada/ grelhada, lingüiça e mortadela, comparando-se ao grupo controle e neste, observou-se maior consumo de iogurte, queijo, hambúrguer e salsicha.

No grupo 2 dos alimentos de origem vegetal, observouse que o grupo dos pacientes com câncer gástrico e o grupo controle apresentou diferença em relação ao consumo de frutas e legumes: os pacientes tiveram menor consumo de frutas e maior consumo de legumes. 
No grupo dos alimentos fontes de gorduras (grupo 3) os pacientes com câncer gástrico apresentaram menor consumo de margarina, gordura vegetal hidrogenada e azeite e maior de toucinho/torresmo/banha em relação ao do grupo controle.

Em relação ao grupo 4, alimentos fonte de carboidratos, observou-se que pacientes com câncer gástrico apresentaram maior consumo de farinha de mandioca, biscoito com e sem recheio, bolo, açúcar refinado e salgados fritos. Apresentou menor consumo de chocolate e de outros tipos de açúcar, quando comparado ao grupo controle.

Analisando-se o grupo 5 dos alimentos utilizados como temperos de preparações, constatou-se que os pacientes com câncer gástrico apresentaram maior consumo de salsa, tempero pronto e glutamato monossódico em relação ao grupo controle.

Em relação ao grupo 6, bebidas alcoólicas, não-alcoólicas e infusões, notou-se que os pacientes com câncer gástrico apresentaram maior consumo de aguardente e refrigerante $\mathrm{e}$ menor consumo de chá, quando comparados ao grupo controle. Os resultados descritos acima estão resumidos no Figura 5.

\begin{tabular}{|c|c|c|}
\hline Grupo de alimentos & Grupo caso & Grupo controle \\
\hline 01 & $\begin{array}{l}\text { ovo, carne bovino frita, } \\
\text { assada e grelhada, lingüiça e } \\
\text { mortadela }\end{array}$ & $\begin{array}{l}\text { iogurte, queijo, hambúrguer } \\
\text { e salsicha }\end{array}$ \\
\hline 02 & legumes & frutas \\
\hline 03 & toucinho, torresmo e banha & $\begin{array}{l}\text { margarina, gordura vegetal } \\
\text { hidrogenada e azeite }\end{array}$ \\
\hline 04 & $\begin{array}{l}\text { farinha de mandioca, biscoito } \\
\text { com e sem recheio, bolo, } \\
\text { açúcar } \\
\text { refinado e salgados fritos }\end{array}$ & $\begin{array}{l}\text { chocolate e outros tipos de } \\
\text { açúcar }\end{array}$ \\
\hline 05 & $\begin{array}{l}\text { salsa, tempero pronto e } \\
\text { glutamato monossódico }\end{array}$ & - \\
\hline 06 & aguardente e refrigerante & chá \\
\hline
\end{tabular}

FIGURA 5. Relação de maior freqüência no consumo de alimentos organizados nos dois grupos

\section{DISCUSSÃO}

Nos últimos 25 anos, tem-se estudado a relação entre o câncer de estômago e a alimentação ${ }^{(2,14,25,30)}$. Segundo informações do Instituto Nacional do Câncer - INCA, o câncer gástrico atinge, em cerca de $65 \%$ dos casos, indivíduos com mais de 50 anos, sendo que o pico de incidência ocorre por volta dos 70 anos de idade, acometendo prioritariamente os homens ${ }^{(14)}$. Em estudo realizado por MADER et al. ${ }^{(13)}$, com 40 pacientes com adenocarcinoma gástrico, observou-se predomínio do sexo masculino $(72,5 \%)$, com média de idade de 61 anos. Neste estudo, cerca de $60 \%$ dos indivíduos foram do sexo masculino e, entre eles, a média de idade foi de aproximadamente 60 anos.

Entre o início dos sintomas, o diagnóstico e os procedimentos instituídos em seu tratamento, os pacientes com câncer apresentaram perda de peso significante. A redução da ingestão de alimentos aparece como um dos fatores que inicia a perda de peso e está associada à perda de apetite, dispepsia, alteração da motilidade intestinal, disfagia, presença do tumor e, ainda, à radioterapia e/ou quimioterapia ${ }^{(11,12)}$. A perda de peso leva à desnutrição e à caquexia e, conseqüentemente, à dificuldade no tratamento e pior prognóstico, além de diminuir significativamente a qualidade de vida ${ }^{(11,27)}$.

No grupo controle foi observada manutenção do sobrepeso com aumento de seu peso corpóreo no decorrer dos anos, que pode ser pelo aumento da idade, uma vez que estudos epidemiológicos mostram que esse aumento tende a ser acompanhado também de aumento de peso. Os doentes com câncer apresentavam sobrepeso antes da doença, no entanto não há um consenso entre obesidade e aumento de risco de câncer gástrico ${ }^{(31)}$.

Observando-se os dados de escolaridade dos indivíduos, verificou-se que no grupo de pacientes, 21 indivíduos nunca ingressaram na escola e dos 49 que estudaram, cerca de $55 \%$ não conseguiram concluir o primeiro grau. Entre os indivíduos do grupo controle, do total, apenas 6 nunca ingressaram na escola e, dos 64 pacientes que estudaram, 27\% não concluíram o primeiro grau.

A correlação inversa entre a prevenção do câncer e a dificuldade de acesso aos estudos é observada não somente em pacientes com câncer gástrico, mas também em indivíduos que apresentam outros tipos de neoplasias ${ }^{(6)}$. Nesse sentido, os profissionais de saúde devem orientar de maneira preventiva a população em geral para tratamento de eventual doença instalada, com uma linguagem de fácil compreensão, para que esse ensinamento seja eficiente ${ }^{(18)}$

Analisando-se as condições de moradia dos indivíduos, verifica-se que, atualmente, todos têm acesso à energia elétrica e saneamento básico; no entanto, no passado, mais da metade dos pacientes com câncer moravam no campo e não tinham acesso a saneamento básico e energia elétrica. Segundo TIAN et al. ${ }^{(27)}$, pacientes moradores de regiões afastadas tendem a ter uma qualidade de vida inferior aos indivíduos que têm acesso aos serviços básicos de saúde, nas comunidades, porque esses têm condições de prevenir o desenvolvimento do câncer ou conseguir tratá-lo em estádio inicial.

Apesar de haver entre os indivíduos do grupo controle o registro do consumo de bebidas alcoólicas e tabagismo, verificou-se maior tempo de consumo de cigarros e maior quantidade de pessoas consumindo álcool entre os doentes com câncer. NISHINO et al. ${ }^{(15)}$, em metanálise realizada em 2006, relataram que a presença de Helicobacter pylori, o consumo de alimentos ricos em sal, associados ao tabagismo, aumenta o risco de câncer gástrico, no entanto não observaram relação entre o consumo moderado de cigarros e câncer gástrico.

Em estudo caso-controle realizado na Sérvia, observouse incidência maior de câncer gástrico entre indivíduos que consumiam leite integral, carne de carneiro, cordeiro e bezerro, açúcar, pão branco e alimentos salgados e, como fatores protetores, margarina, queijos não-amarelos e peixes ${ }^{(23)}$. Os doentes desta casuística consumiam menos iogurte e queijo, em relação ao grupo controle; e, em relação aos ovos, 32,8\% dos pacientes os consumiam com maior freqüência que o grupo controle. Não houve diferença entre os dois grupos, quando comparado o consumo de carne de ave assada, grelhada ou frita. Ambos apresentavam consumo médio de 2 a 3 vezes por semana, assim 
como no consumo de carne suína. Quanto ao consumo de peixes, também não foi observada diferença entre os dois grupos; no entanto, observou-se que o hábito de ingestão desse alimento é pouco comum entre os indivíduos. A maioria relatou que só mensalmente, ou nunca, consumia esse alimento.

Independente da forma de preparo, tanto frita como assada ou grelhada, os pacientes com câncer gástrico consumiram mais carne bovina, além de embutidos como lingüiça, salsicha, presunto, mortadela e salame, do que indivíduos do grupo controle. Em relação ao item churrasco, não houve diferença. O consumo excessivo de carnes é um fator que está relacionado com o risco de desenvolvimento de vários tipos de câncer, como o colorretal, o de mama e o de próstata. A Europa vem pesquisando, desde 2002, a relação entre alimentos e carcinogênese (EPIC - European Prospective Investigation Into Cancer and Nutrition) e constatou relação positiva entre alto consumo de carne bovina ou carne processada e incidência de adenocarcinoma gástrico e de esôfago. Pouca relação foi observada entre indivíduos que consumiam carne de ave e carne processada, e moderada relação entre aqueles que consumiam carne bovina e de aves.

Tanto os pacientes com câncer gástrico quanto os indivíduos do grupo controle referiram consumo freqüente de verduras cruas e cozidas. Indivíduos de ambos os grupos consumiam frutas mais de 4 vezes por semana; no entanto, os pacientes com câncer consumiam mais legumes cozidos do que o grupo controle. O consumo de vegetais crucíferos, que apresentam flores em formato de cruz, como o brócolis, a couve-flor, a couve-de-bruxelas e o repolho, tem a capacidade de destoxificar carcinógenos, oferecendo proteção ao organismo humano contra o desenvolvimento de alguns tipos de câncer, como o de próstata e cólon ${ }^{(20)}$.

Questão importante a ser considerada é que alguns vegetais são consumidos habitualmente crus, enquanto outros são cozidos. A comparação, no entanto, fica prejudicada, já que não são os mesmos. Além disso, há uma variação no tamanho da porção consumida entre vegetais crus e cozidos, dificultando a interpretação dos resultados.

Estudos evidenciam que o consumo de vegetais e frutas cítricas exerce fator protetor e conseqüente redução na incidência dessa neoplasia ${ }^{(5,8)}$. Em estudo experimental realizado em ratos com adenocarcinoma gástrico, após a retirada do tumor por procedimento cirúrgico e ofertas diárias de vitamina $\mathrm{C}$, observouse que não houve recidiva da neoplasia, sugerindo efeito protetor da vitamina. Esta ação deve-se, provavelmente, à propriedade antioxidante desta vitamina e a habilidade de reagir com nitritos, prevenindo a formação de compostos N-nitrosos ${ }^{(17)}$.

Alimentos fontes de amido como arroz, tubérculos (mandioca, mandioquinha, batata, cará, entre outros), pão branco entre outros, e carnes processadas, são relacionados com o desenvolvimento de câncer gástrico, enquanto alimentos como vegetais frescos e frutas são considerados protetores ${ }^{(4)}$.

O consumo de margarina, gordura vegetal hidrogenada e azeite de oliva foi menor entre os pacientes com câncer. $\mathrm{O}$ azeite de oliva tem sido relacionado à prevenção de vários tipos de câncer, mas ainda com mecanismo não definido. Sugere-se que possa existir relação entre a presença de ácidos graxos monoinsaturados na regulação específica de oncogenes ${ }^{(3)}$.

Farinha de mandioca, biscoitos recheados e sem recheio, açúcar branco e salgados fritos (como coxinha, quibe, pastel, etc) foram consumidos com maior freqüência pelos pacientes com câncer, porém não houve diferença no consumo de arroz, feijão e macarrão entre os grupos.

TSUGANE(28) relata que não há relação entre o consumo de alimentos conservados em sal, o sal propriamente dito, e o câncer gástrico. No entanto, a maioria dos autores mostra que doentes com câncer apresentavam maior consumo de sal em relação ao grupo controle e que a presença da bactéria Helicobacter pylori aumentava significantemente a incidência desse tipo de câncer.

Os vegetais do gênero Allium, como o alho, alho poró, cebola, cebolinha-verde, chalota e cebolinha contêm alicina, que exerce ação antimutagênica, que estaria relacionada à proteção ao câncer gástrico ${ }^{(7)}$. Além disso, verificou-se que extrato de alho possui poder bactericida, in vitro, contra o Helicobacter pylori ${ }^{(24)}$.

Neste estudo não foi possível encontrar diferenças entre o consumo de sal, alho e cebola entre os indivíduos dos dois grupos; no entanto, observou-se que os pacientes com câncer faziam maior uso de tempero pronto (caldos de carnes, legumes, etc) e glutamato monossódico.

Pôde-se observar, através deste estudo, que os pacientes com câncer gástrico apresentaram características de vida inferiores aos indivíduos do grupo controle, no que diz respeito a acesso à educação escolar, saneamento básico, acesso à energia elétrica e moradia no passado. Verificou-se, também, que houve diferença de consumo de alguns alimentos nos dois grupos, sendo que o grupo de pacientes apresentou maior consumo de ovos, gemada, carne bovina frita, assada e grelhada, lingüiça, legumes, farinha de mandioca, biscoito recheado e sem recheio, bolo, açúcar refinado, salgados fritos, salsa, tempero industrializado e glutamato monossódico, enquanto os indivíduos do grupo controle apresentaram maior consumo de iogurte, queijo, hambúrguer, salsicha, frutas, margarina, gordura vegetal hidrogenada, azeite, chocolate, açúcar de outros tipos que não o refinado e chá. 
Magalhães LP, Oshima CTF, Souza LG, Lima JM, Carvalho L, Forones NM. Weight, educational achievement, basic sanitation, alcoholism, smoking and eating habit in patients with gastric cancer. Arq Gastroenterol. 2008;45(2):111-6.

ABSTRACT - Background - About 35\% of the cancer patients are involved in factors coming from the diet and others like alcohol, smoking, sunlight, chemical agents and infections caused by virus. The stomach cancer is the second cause of cancer in the world with $9.9 \%$ of all diagnosis and about $12.1 \%$ of death cases. Aims - Evaluate the body weight, educational achievement, basic sanitation, smoking, alcoholism and eating habit among patients with gastric cancer and a control group. Methods - Seventy patients with gastric cancer were paired with 70 subjects without cancer. Data on the weight and height, educational attainment, basic sanitation, smoking, alcoholism and eating habits of the patients were collected from the clinical records and from interviews. Results - Forty two patients were men, the mean age were 60 years old. The actual weight and body mass index of the patients were smaller when compared to the controls. Within the group of patients with gastric cancer, 21 never attended school, and for those who attended, 55\% did not finish the elementary school. Among the patient group, $32.9 \%$ of them lived in housing with basic sanitation and $37.1 \%$ with electricity, against $68.6 \%$ of the controls, and $58.6 \%$ of the patients lived in rural area, against only $7.1 \%$ of the controls. Among the test group, $65.7 \%$ of the patients were smokers, whereas in the control group, $44.3 \%$ were smokers. In addition, there was also difference in the duration of smoking habit. Alcoholism was also more frequent in the cancer group (44\% vs $19 \%$ ). Food rich in salt, condiments, nitrates, saturated fat, complex carbohydrates, refined sugar and fried salted food had been more used by gastric cancer patients. Conclusion - The patients with gastric cancer presented with: less weight, low quality of life as indicated by lower or no access to basic sanitation, electricity and schooling, lived predominantly in rural area, high incidence of alcohol intake and higher intake of high fat foods and industrialized foods.

HEADINGS - Stomach neoplasms. Body weight changes. Educational status. Basic sanitation. Alcoholism. Smoking. Food habits.

\section{REFERÊNCIAS}

1. Bresciani C, Ribeiro Jr U, Ribeiro AVS, Perez RO, Jacob CE, Zilberstein B, GamaRodrigues J. Dieta e câncer gástrico. In: Waitzberg DL, editor. Dieta, nutrição e câncer. São Paulo: Atheneu; 2004. p.238-42.

2. Cecconello I, Leite AF. Influência da dieta na gênese do câncer de esôfago. In: Waitzberg DL, editor. Dieta, nutrição e câncer. São Paulo: Atheneu; 2004. p.243-6.

3. Colomer R, Menendez JA. Mediterranean diet, olive oil and cancer. Clin Trans Oncol. 2006;8:15-21.

4. De Stefani E, Correa P, Boffetta P, Deneo-Pellegrini H, Ronco AL, Mendilaharsu M. Dietary patterns and risk of gastric cancer: a case-control study in Uruguay. Gastric Cancer. 2004; 7:211-20.

5. Erickson KL. Dietary pattern analysis: a different approach to analyzing an old problem, cancer of the esophagus and stomach. Am J Clin Nutr. 2002;75:5-7.

6. Fujino Y, Tamakoshi A, Ohno Y, Mizoue T, Tokui N, Yoshimura T, JACC Study Group, Japan Colaborative Cohort study for evaluation of cancer risk. Prospective study of educational background and stomach cancer in Japan. Prev Med. 2002;35:121-7.

7. Gao C, Takezaki T, Wu J, Li Z, Wang J, Ding J, Liu Y, Hu X, Xu T, Tajima K Sugimura $\mathrm{H}$. Interaction between cytochrome P-450 2E1 polymorphisms and environmental factors with risk of esophageal and stomach cancers in Chinese. Cancer Epidemiol Biomarkers Prev. 2002;11:29-34.

8. González CA, Jakszyn P, Pera G, Agudo A, Bingham S, Palli D, Ferrari P, Boeing H, del Giudice G, Plebani M, Carneiro F, Nesi G, Berrino F, Sacerdote C, Tumino R, Panico S, Berglund G, Simán H, Nyrén O, Hallmans G, Martinez C, Dorronsoro M, Barricarte A, Navarro C, Quirós JR, Allen N, Key TJ, Day NE, Linseisen J, Nagel G, Bergmann MM, Overvad K, Jensen MK, Tjonneland A, Olsen A, Bueno-de-Mesquita HB, Ocke M, Peeters PH, Numans ME, Clavel-Chapelon F, Boutron-Ruault MC, Trichopoulou A, Psaltopoulou T, Roukos D, Lund E, Hemon B, Kaaks R, Norat T, Riboli E. Meat intake and risk of stomach and esophageal adenocarcinoma within the European Prospective Investigation Into Cancer and Nutrition (EPIC). J Natl Cancer Inst. 2006;98:345-54.

9. Gotay CC. Behavior and cancer prevention. J Clin Oncol. 2005;23:301-10.

10. Laranjeira R, Pinsky I. Alcoolismo. São Paulo: Contexto; 1998.

11. Liedman B. Symptoms after total gastrectomy on food intake, body composition, bone metabolism, and quality of life in gastric cancer patients - is reconstruction with a reservoir worthwhile? Nutrition. 1999;15:677-82.

12. Lim L, Michael M, Mann GB, Leong T. Adjuvant therapy in gastric cancer. J Clin Oncol. 2005:23:6220-32.

13. Mader AM, Patrício FR, Rigueiro MP, Lourenço LG. Estudo clínico-patológico, da proliferação celular e da apoptose no adenocarcinoma gástrico da cárdia. Arq Gastroenterol. 2006;43:184-90.

14. Ministério da Saúde. INCA - Instituto Nacional de Câncer [sítio na Internet]. Apresenta texto sobre câncer de estômago; 1996. [citado 2006 jul 18]. Disponível em: URL: http://www.inca.gov.br/
15. Nishino Y, Inoue M, Tsuji I, Wakai K, Nagata C, Mizoue T, Tanaka K, Tsugane S, Research Group for the Development and Evaluation of Cancer Prevention Strategies in Japan. Tobacco smoking and gastric cancer risk: an evaluation based on a systematic review of epidemiologic evidence among the Japanese population. Jpn J Clin Oncol 2006;36:800-7.

16. Norman HA, Butrus RR. Dieta, nutrição e câncer: direções para pesquisas futuras. In: Waitzberg DL, editor. Dieta, nutrição e câncer. São Paulo: Atheneu; 2004. p.647-52.

17. Oliveira CP, Kassab P, Lopasso FP, Souza HP, Janiszewski M, Laurindo FR, Irya K, Laudanna AA. Protective effect of ascorbic acid in experimental gastric cancer: reduction of oxidative stress. World J Gastroenterol. 2003;9:446-8.

18. Pohls UG, Fasching PA, Beck H, Kaufmann M, Kiechle M, von Minckwitz G, Paepke S, Schultz-Zehden B, Schwarz-Boeger U, Beckmann MW. Demographic and psychosocial factors associated with risk perception for breast cancer. Oncol Rep 2005; $14: 1605-13$

19. Qiu JL, Chen K, Zheng JN, Wang JY, Zhang LJ, Sui LM. Nutritional factors and gastric cancer in Zhoushan Islands, China. World J Gastroenterol. 2005:11:4311-6.

20. Rose P, Ong CN, Whiteman M. Protective effects of Asian green vegetables against oxidant induced cytotoxicity. World J Gastroenterol. 2005;11:7607-14.

21. Rosner B. Fundamentals of biostatistics. 2nd ed. Boston: PWS; 1986. 584p

22. Sachs A. Hábito alimentar e estado nutricional de pacientes idosos ambulatoriais, pré e pós-diagnóstico de doença pulmonar obstrutiva crônica [tese]. São Paulo: Universidade Federal de São Paulo; 1997.

23. Sipetic S, Tomic-Kundakovic S, Vlajinac H, Jankovic S, Marinkovic J, Maksimovic J. Diet and gastric cancer. Vojnosanit Pregl 2003;60:697-705.

24. Sivam GP, Lampe JW, Ulness B, Swanzy SR, Potter JD. Helicobacter pylori-in vitro susceptibility to garlic (Alliun sativum) extract. Nutr cancer. 1997;27:118-21.

25. Strumylaitè L, Ži kutè J, Dudzevi ius J, Dregval L. Salt-preserved foods and risk of gastric cancer. Medicina (Kaunas) 2006;42:164-70.

26. Taylor PL, Greenwald P. Nutritional Interventions in cancer prevention. J Clin Oncol. 2005;23:333-45.

27. Tian J, Chen JS. Nutritional status and quality of life of the gastric cancer patients in Changle County of China. World J Gastroenterol. 2005;11:1582-6.

28. Tsugane S. Salt, salted food intake, and risk of gastric cancer: epidemiologic evidence Cancer Sci. 2005;96:1-6.

29. World Health Organization [sítio na Internet]. Apresenta texto sobre câncer. [citado 2006 jul 10]. Disponível em: URL: http://www.who.int/cancer/en/.

30. Ye WM, Yi YN, Luo RX, Zhou TS, Lin RT, Chen GD. Diet and gastric cancer: case-control study in Fujian Province, China. World J Gastroenterol. 1998:4:516-8.

31. Zhang J, Su XQ, WU XJ, Liu YH, Wang H, Zong XN, Wang Y, Ji JF. Effect of body mass index on adenocarcinoma of gastric cardia. World J Gastroenterol. 2003;9:2658-61.

Recebido em 2/7/2007 Aprovado em 9/10/2007. 